lung had become aerated. The lower part still showed a dense shadow, and the heart and mediastinum were still over on the right side. The right border of the heart could be made out more distinctly, and the trachea could be seen to be over on the right side (Fig. 2). Physical examination revealed tympany on percussion over the upper two right interspaces, with mixed bronchial and vesicular breathing, and many râles. For the next two days the physical signs remained the same. The dyspnea was much less, and there was no abnormal temperature. April 22, the picture had changed entirely. The apex beat was felt distinctly in the fifth interspace just inside the nipple line, and the right border of the heart could be made out on percussion, just outside the right border of the sternum. The breath sounds were heard distinctly over the whole right lung anteriorly, and posteriorly with numerous râles. The collapsed lung had again become inflated, and the heart and mediastinum were back in their normal position. The roentgenogram taken on the 24th showed the heart and mediastinum in their normal position. The lower part of the right lung was still not quite aerated. The roentgenogram taken on the 26th showed the same picture (Fig. 3). There were still some râles heard at the right base. A roentgenogram taken, May 10 , revealed the heart and mediastinum in normal position and both lungs entirely clear (Fig. 4). Physical examination on the same date was negative.

\section{COMMENT}

This case undoubtedly represents one of massive collapse of the right lung following a stab wound in the abdomen. What part the wound itself and what part the anesthesia played in the production of the collapse cannot be determined. As we become more familiar with this syndrome, we shall probably find that many cases of postoperative pneumonia will prove to be massive collapse of the lung.

How the collapse is produced, we do not know. Pasteur assumes that the collapse of the lung following operation is due to a reflex inhibition of the movements of the diaphragm. He showed that paralysis of the diaphragm and chest wall, if persistent for two days, results in collapse of the lung. Elliott and Dingley have assumed the condition to be due to obstruction of the bronchial tubes by secretion. At necropsy no such obstruction is found. Briscoe assumes that, as a result of inflammation of the diaphragm and of the pleural membrane covering it, the diaphragm and its synergic and antagonistic muscles are out of action, and that this results in massive collapse of the lung-an opinion not much different from that of Pasteur. Perhaps: experimental studies will eventually solve this problem.

141 West One Hundred and Tenth Street-204 West One Hundred and Tenth Street.

Cancer a Local Disease.-As cancer is a local disease, there is always a stage when complete removal and cure is possible. Successful surgical treatment then is a matter of early removal.-Wolbach, New Growths and Cancer, Harvard University Press, 1922.

\title{
FIBROSIS OF THE LUNG FOLLOWING ROENTGEN-RAY TREATMENTS FOR TUMOR
}

\author{
LAURENCE E. HINES, M.D. \\ CHICAGO
}

There is little known about the effect of the roentgen ray on lung tissue as applied in the treatment of pulmonary neoplasms. Holmes, ${ }^{1}$ in summarizing the present status of roentgen-ray therapy, emphasizes that investigation is necessary to determine the amount and quality of irradiation that should be used in different diseases, the effect of irradiation on both normal and pathologic tissues, and the effect of treatment on the patient as a whole. The present report of the lesions in two cases of secondary lung tumors which were treated with roentgen rays may throw some light on the effect of irradiation on the normal lung and on tumors of the lungs.

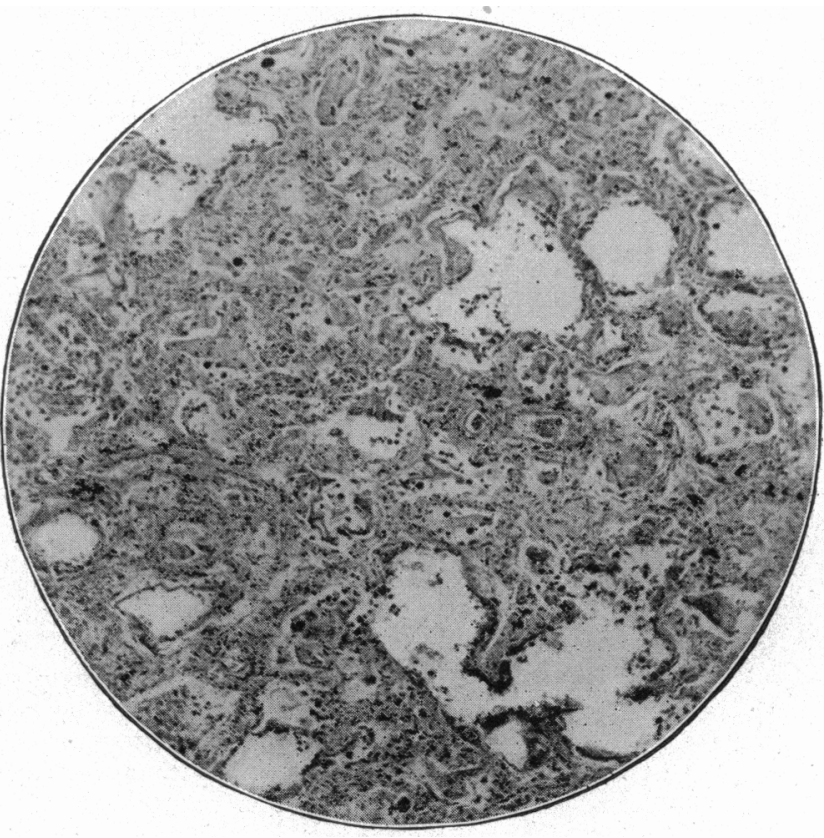
Fig. 1.-Low power of section of lung from Case 1, showing extent phrenic angle was obliterated; the right diaphragm could not be outlined; in the left lung near the vertebra, at the level of the sixth rib posteriorly, there was a circumscribed nodule, and there were many large nodules in the right lower lobe.

Trcatment and Course.--Three series of roentgen-ray treatments were given under the direction of Dr. Jenkinson: Oct. 5, 1921, Series 1, 140,000 Peak volts at 20 inches target distance from the skin, with a filter of $1 \mathrm{~mm}$. of copper and $1 \mathrm{~mm}$. of aluminum; 2,610 milliampere minutes were given over all parts of the chest; November 17, Series 2, 125,000 Peak volts at 12 inches target distance from the skin, with a filter of $10 \mathrm{~mm}$. of aluminum; 2,400 milliampere minutes over the entire chest; Feb. 2, 1922, Series 3, 140,000 Peak volts at 20 inches target distance from the skin with a filter of $1 \mathrm{~mm}$. of aluminum and $1 \mathrm{~mm}$. of copper ; 1,500 milliampere minutes over the front of the chest and 900 over the back of the chest were given. No further treatments were given because the patient's condition became worse.

After the first series, improvement was almost immediate, the cough improved, and he gained in weight and strength. The third series was interrupted because the cough leecame worse and dyspnea developed. March 3, the respiratory dis-

\footnotetext{
1. Inlmes. G. W.: Am. J. Roentgenol. 8:522 (Sept.) 1921.
} 
tress was so pronounced that he was admitted to Dr. Frederickson's service in St. Joseph's Hospital. He was mildly cyanotic, and complained of air hunger. Chest expansion was equal on the two sides and not limited. A general decrease in lung resonance and moderate dulness over the right lower lobe was present. There was no enlargement of the heart, nor were any heart murmurs audible. The systolic blood pressure was 120 , and the diastolic 70 . Repeated bacteriologic examinations of the sputum revealed pneumococci and streptococci, but no tubercle bacilli. Sometimes the sputum was mucopurulent, at other times of tenacious, mucoid consistency. The hemoglobin estimation was 74 per cent. by the Sahli scale; there were $4,000,000$ erythrocytes and 5,600 leukocytes per cubic millimeter. The urine and feces showed nothing abnormal. The patient had a fairly constant fever varying from 100 to 101 . The breathing was shallow and at a rate of from 30 to 50 a minute. The pulse was of good quality, not more than 100 a minute. He frequently complained of an inability to get sufficient air. The breathing became more and more difficult, the cyanosis deepened, and on March 13 he died.

Necropsy. - At the postmortem examination, af)out six hours after death, the body was that of a well developed man, $170 \mathrm{~cm}$. long, weighing about 170 pounds (77 kg.) The nourishment was good. The skin and the mucous membranes were moderately cyanotic. The left elbow joint was anl:ylosed by a bony union. Two old scars extended vertically along the lateral surface of the left arm. There was a palpable thickening of the middle third of the left humerus, adherent to the scars. The humerus was removed and a fusiform enlargement of the middle, $4 \mathrm{~cm}$. in diameter at the thickest portion, was composed of a soft, gray, friable growth that originated from the periosteum.

The anterior and superior mediastinal areolar tissue was thick and tough.

In each pleural cavity there was not more than 50 c.c. of clear, straw-colored fluid. Dense fibrous adhesions extended between the lower lobe of the right lung and the pos-

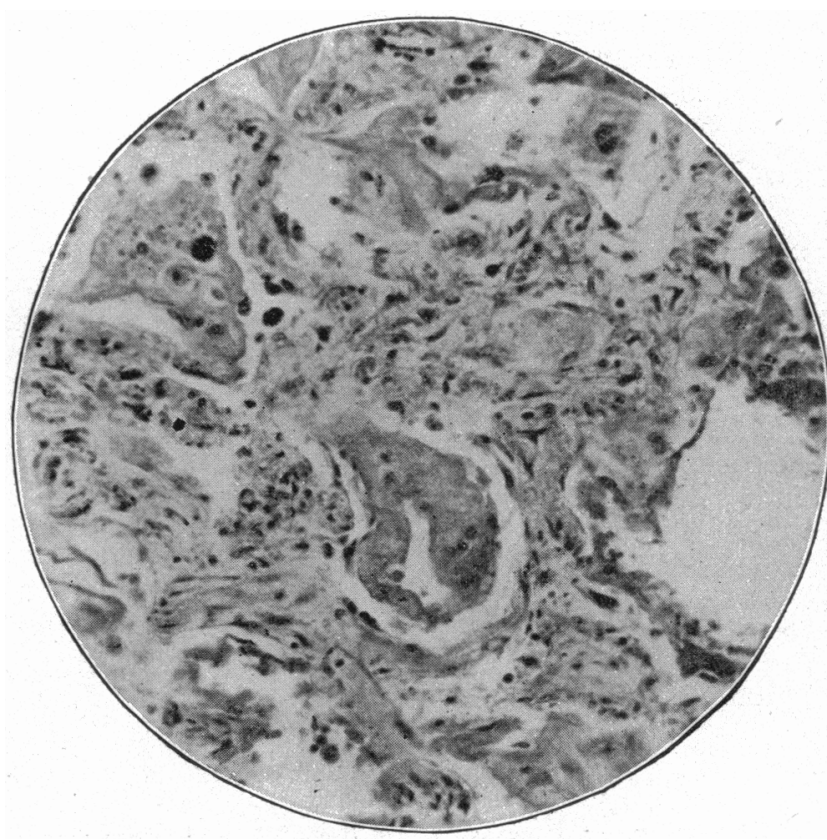

Fig. 2.- High power of section of lung from Case 1, showing proFig. 2.- - High power of section of lung fr
liferation of fibroblasts in the alveolar walls.
Except for slight fibrous thickening of the leaflets of the mitral valve, there was no change in the heart. The spleen was about twice its normal size, the capsule thickened and fibrous markings prominent.

Microscopic examination of sections from different parts of the tumor about the left humerus and from the nodules in the lung revealed a sarcoma with a predominance of large spindle cells of uniform size and appearance, arranged in twisted bundles. At the site of the tumor in the arm, the tissue was more cellular, with only a small amount of fibrillar intercellular substance, while in the lung tumor the intercellular substance was more prominent and contained abundant young and old connective tissue cells. The large spindle cells predominating in the sarcoma had a narrow rim of homogeneous protoplasm with large nuclei. Mitotic figures were infrequent.

Sections made from various parts of both lungs showed a decrease in the size of the alveoli and total obliteration of many of them, a striking feature, due to an increase in the alveolar walls by proliferation of fibroblasts, some of which extended into the lumen. There was a small amount of round-cell infiltration. In some of the alveoli, coagulum (edema) was present; in others, desquamatetd alveolar epithelium. The obliterated alveoli were filled with almost solid connective tissue. There was also some hyperplasia of the perivascular and peribronchial connective tissue, and a proliferation of connective tissue in the mediastinal lymph glands. The fibrous tissue in the spleen was increased.

CAse 2.-History.-A white woman, aged 53 , was admitted to St. Joseph's Hospital, April 20, 1922, complaining of severe dyspnea, orthopnea and cyanosis. She lived only eight hours. In 1913, the right breast had been amputated because of a tumor. In July, 1921, enlarged right axillary glands were removed, and microscopic examination revealed the presence of closely packed columns and strands of carcinoma.

Following the operation, under the direction of $\mathrm{Dr}$. H. E. Potter, she was given four series of roentgen-ray treatterior wall and diaphragm. There were no adhesions in the left pleural cavity. The left lung weighed about $1,000 \mathrm{gm}$; the surface was smooth; crepitation was absent in all portions; the substance was tough on section, and the cut surface had a dry, liver-like appearance. Multiple sections through all parts of both lobes revealed a lung substance of uniform dark brown appearance with but little air-containing tissue. No pus could be expressed from the bronchi, and no fibrin plugs were visible in the small amount of blood-stained fluid that was scraped from the cut surface. The right lung weighed about $1,100 \mathrm{gm}$. Except for a single nodule, $5 \mathrm{~cm}$. in diameter, the upper lobe of the right lung was similar in all respects to the left lung. The lower and middle lobes of the right lung were densely adherent to each other by fibrous adhesions. Tumor tissue had replaced more than half of these lobes. There were several round gray-white, shining, circumscribed nodules averaging $2 \mathrm{~cm}$. in diameter, closely set together and separated by a small amount of lung tissue similar in appearance to the parenchyma of the left lung. There was slight edema of the peritracheal and peribronchial lymph nodes. The lymph nodes in the superior mediastinum were enlarged, tough and surrounded by a thick fibrous capsule. ments, in which three areas were exposed each time. A voltage of 85,000 was used, and not more than 200 milliampere minutes were given over each area. In December, 1921, she was also exposed to $500 \mathrm{mg}$. hours of radium over the right supraclavicular region, $600 \mathrm{mg}$. hours in the right axilla and two needles of radium in the right axilla for three hours. In the first part of February, 1922, dyspnea developed and gradually increased in severity. The attending physician diagnosed left pleural effusion, and aspirated 1,000 c.c. of serosanguineous fluid, cultures of which were sterile. The dyspnea, which was only partially relieved by the aspiration, became more severe until her admission to St. Joseph's Hospital. The report of the roentgenogram taken a few hours before her death was: "The left lung is collapsed and fluid is present in the left base. There are circumscribed nodules in the left lung which might be interpreted as being carcinomatous nodules. The right lung is practically solid."

Necropsy.-At the postmortem, twelve hours after death, the body was $167 \mathrm{~cm}$. long, weighing about 130 pounds (59 $\mathrm{kg}$.). Nourishment was good, and there was an abundance of subcutaneous fat. The right breast was absent, and an old scar extended from the right anterior chest wall to and around the axillary fold. There were no nodules in the scar 
or adjacent tissue. The subcutaneous tissue in the region of the scar was tightly bound to the chest wall by fibrous adhesions. No tumor tissue was found in the axilla or regional lymph nodes. There was about 1 liter of bloodstained fluid in the left pleural cavity. Many strands of easily torn vascular adhesions extended from the left lung to the posterior parietal pleura. There was about 1 liter of clear straw-colored fluid in the right pleural cavity, but no adhesions. Both right and left parietal pleurae were increased in thickness to $5 \mathrm{~mm}$., owing to fibrous and hyaline changes. On the pleural surface of both lungs were irregularly shaped gray nodules averaging $10 \mathrm{~mm}$. in diameter. Dense fibrous adhesions bound the left lung to the diaphragm, and obliterated the left interlobar fissure. The pleura over the posterior and diaphragmatic surfaces of the left lung was thick, grayish white, and of a tough consistency. Both lungs were decreased in size, the right one weighing $650 \mathrm{gm}$., and the left one, $725 \mathrm{gm}$. Three gray nodules averaging $15 \mathrm{~mm}$. in diameter were found in the right lung. No nodules were found in the left lung. Both lungs were of a leathery consistency and noncrepitant. The lung substance had a uniform fleshy appearance, and dark, bloody fluid exuded from the cut surface. There was no pus in the bronchi or bronchioles, nor were any fibrin plugs found after squeezing the lung.

In the liver there was one nodule of tumor tissue $2 \mathrm{~cm}$. in diameter. The myocardium was pale, and a few yellow plaques were seen about the mouths of the coronary arteries and in the ascending aorta. No notable changes were observed in the other viscera.

Microscopic examination of the tumor nodules in the lung revealed closely packed columns and strands of carcinoma cells with a fine stroma. The cells were of varying ovoid shapes, the nuclei large and granular, and with frequent mitotic figures. Degenerated areas were frequent. In the liver was an area in which the liver cells were almost completely replaced by carcinoma. The parietal pleura was a dense, fibrous tissue with hyaline deposits. The friable adhesions in the left pleural cavity were composed of hemorrhagic fibrinous material.

Islands of carcinoma had invaded all parts of both lungs, the islands being sharply demarcated from the surrounding tissue by a dense fibrous capsule. The alveolar walls were slightly thickened and compressed with resultant diminution of air space, the thickening due chiefly to edema, hyperemia, and small round-cell infiltrations, but there were some connective tissue cells present.

\section{COMMENT}

In Case 1, a spindle-cell sarcoma with lung metastasis, roentgen-ray treatment was given according to the newer method of higher voltage, greater filtration and longer target distance. At first there was improvement in the patient's condition; but, during the last series of treatments, symptoms of respiratory distress developed, and several days later death occurred. All parts of both lungs, postmortem, were consolidated from proliferation of connective tissue in the alveolar wall, with resultant great decrease in air space, which accounts for the dyspnea and cyanosis, and might be considered as the immediate cause of death. In Case 2, carcinoma of the breast with pleural and lung metastasis, only a limited amount of roentgen-ray treatment was given, and no large amount at any one time. As in Case 1, cyanosis and dyspnea were prominent symptoms, and the gross appearance of the lungs was similar in the two cases; but microscopic examination disclosed that the consolidation was due to a diffuse infiltration of the lung tissue with islands of carcinoma. Therefore, the dyspnea and cyanosis and the cause of death may be attributed to the extensive growth of tumor in the lungs. However, the hyperplasia of connective tissue about the islands of carcinoma in the lung may have been the result, at least in part, of irradiation.

Numerous reports of the beneficial effects on deep seated tumors from roentgen-ray treatment give the impression that irradiation may inhibit the growth or destroy it without harmful effect on neighboring normal tissue. Thus, Soiland, ${ }^{2}$ though he admits that it is logical to assume that rays passing through normal and pathologic cells should exert a like effect on each, states that there is little clinical evidence that normal tissues are injured by deep roentgen-ray therapy. In Case 1 , in which heavy doses were used, it is difficult to explain the condition found except as the result of the irradiation.

\section{SUMMARY}

The condition of the lungs in a case of secondary sarcoma indicates that normal lung tissue may become the seat of a pathologic fibrosis as the result of intensive roentgenization.

In a case of secondary carcinoma of the lungs, treated less intensively, extensive infiltrations were encapsulated by fibrous tissue.

St. Joseph's Hospital.

\section{CONDENSED AND ACCURATE CHARTING \\ OF THE DIAGNOSIS OF THE EAR, NOSE, THROAT AND TEETH*}

W. PERRY REAVES, M.D.

GREENSBORO, N. C.

Records are of value in proportion to their etiologic accuracy, their accessibility and their legibility. The lower half of the head contains, in a comparatively small area, the upper respiratory tract, the beginning of the digestive tract and four of the special senses. Anatomically and physiologically, each two seem to be codependent. The eye needs the lacrimal duct for drainage into the nose; the ear the eustachian tube for ventilation. The olfactory bulbs function best in a well-ventilated nose. The gustatory sense is aided by the olfactory sense. This territory has been divided into sections or states of ophthalmology, otology, rhinology, laryngology and dentistry. The fifth nerve and its ganglions seem to be in high command and often bind them together in pain. An outbreak of the pathologic conditions or focal infection in one section appears to have a selected affinity, by continuity or contiguity of tissue, for its sister state.

The oculist may find an abscessed tooth the cause of choroiditis or iritis, or a sinusitis the cause of an optic neuritis or a labyrinthitis the cause of a nystagmus. The otologist is kept on the defensive to prevent the invasion of the enemy through the eustachian tube. In dentistry, we have thirty-two teeth which may harbor infection at some time in life. In rhinology, we have a good example of reciprocity. The nose and its accessory sinuses may be involved by the continuity and contiguity of tissue of the eye, ear, throat or teeth, and they seem willing at any time to reciprocate its pathologic condition and infection with the eye, ear and throat. The laryngologist may find local trouble resulting primarily or secondarily from reflexes or irritation

2. Soiland, A.: Am. J. Roentgenol. 8:276 (May) 1921

* Read before the Section on Laryngology, Otology and Rhinology at the Seventy.Third Annual Session of the American Medical Association, St. Louis, May, 1922. 